PAPER

Experimental estimation of tungsten impurity sputtering due to Type I ELMs in JET-ITER-like wall using pedestal electron cyclotron emission and target Langmuir probe measurements

To cite this article: C Guillemaut et al 2016 Phys. Scr. 2016014005

View the article online for updates and enhancements.
Related content

\begin{tabular}{l} 
- Ion target impact energy during Type I \\
$\frac{\text { edge localized modes in JET ITER-like }}{\text { Wall }}$ \\
$\frac{\text { C Guillemaut, A Jardin, J Horacek et al. }}{\text { - Intra-ELM tungsten sputtering in JET }}$ \\
$\frac{\text { ITER-like Wall: analytical studies of Be }}{\text { impurity and ELM type influence }}$ \\
\hline I Borodkina, D Borodin, S Brezinsek et al. \\
- Experimental validation of an analytical \\
kinetic model for edge-localized modes in \\
JET-ITER-like wall \\
C. Guillemaut, C. Metzger, D. Moulton et \\
al.
\end{tabular}

Recent citations

$$
\begin{aligned}
& \text { - Deposition in the tungsten divertor during } \\
& \frac{\text { the } 2011-2016 \text { campaigns in JET with }}{\text { ITER-like wall }} \\
& \hline \text { N Catarino et al } \\
& \text { - Estimation of ELM effects on Be and W } \\
& \frac{\text { erosion at JET-ILW }}{\text { I Borodkina } \text { et al }} \\
& \text { - Modelling of the effect of ELMs on fuel } \\
& \text { retention at the bulk W divertor of JET } \\
& \text { K. Heinola et al }
\end{aligned}
$$




\title{
Experimental estimation of tungsten impurity sputtering due to Type I ELMs in JET-ITER-like wall using pedestal electron cyclotron emission and target Langmuir probe measurements
}

\author{
C Guillemaut ${ }^{1,2}$, A Jardin ${ }^{3}$, J Horacek ${ }^{4}$, I Borodkina ${ }^{5}$, A Autricque ${ }^{3}$, \\ G Arnoux ${ }^{6}$, J Boom ${ }^{7}$, S Brezinsek ${ }^{8}$, J W Coenen ${ }^{8}$, E De La Luna ${ }^{9}$, \\ S Devaux ${ }^{10}$, T Eich $^{7}$, D Harting ${ }^{6}$, A Kirschner ${ }^{8}$, B Lipschultz ${ }^{11}$, \\ G F Matthews ${ }^{6}$, A Meigs ${ }^{6}$, D Moulton ${ }^{6}$, M O'Mullane $^{12}$, M Stamp $^{6}$ and JET \\ contributors $^{13}$ \\ ${ }^{1}$ EUROfusion Consortium, JET, Culham Science Centre, Abingdon, OX14 3DB, UK \\ ${ }^{2}$ Instituto de Plasmas e Fusão Nuclear, Instituto Superior Técnico, Universidade Lisboa, PT \\ ${ }^{3}$ CEA, IRFM, F-13 108 Saint-Paul-lez-Durance, France \\ ${ }^{4}$ IPP.CR, Institute of Plasma Physics AS CR, Za Slovankou 3, 18221 Praha 8, Czech Republic \\ ${ }^{5}$ National Research Nuclear University MEPhI, Kashirskoye sh., 31, Moscow, Russia, 115409 \\ ${ }^{6}$ CCFE, Culham Science Centre, Abingdon OX14 3DB, UK \\ ${ }^{7}$ Max-Planck-Institut fur Plasmaphysik, Boltzmannstr. 2, D-85748 Garching, Germany \\ ${ }^{8}$ Instiut für Energie und Klimaforschung-Plasmaphysik, Forschungszentrum Jülich, 52425 Jülich, \\ Germany \\ ${ }^{9}$ Laboratorio Nacional de Fusión,CIEMAT, 28040 Madrid, Spain \\ ${ }^{10}$ Institut Jean Lamour, UMR7198 CNRS-Université de Lorraine, F-54506 Vandoeuvre-les-Nancy \\ Cedex, France \\ ${ }^{11}$ YPI, University of York, York YO10 5DQ, UK \\ ${ }^{12}$ Department of Physics, University of Strathclyde, Glasgow G4 0NG, UK
}

E-mail: christophe.guillemaut@ccfe.ac.uk and cguillemaut@ipfn.ist.utl.pt

Received 28 May 2015, revised 20 August 2015

Accepted for publication 18 September 2015

Published 8 January 2016

\begin{abstract}

\footnotetext{
${ }^{13}$ See the Appendix of F Romanelli et al Proceedings of the 25th IAEA Fusion Energy Conference 2014, Saint Petersburg, Russia
}

The ITER baseline scenario, with $500 \mathrm{MW}$ of DT fusion power and Q $=10$, will rely on a Type I ELMy H-mode and will be achieved with a tungsten (W) divertor. W atoms sputtered from divertor targets during mitigated ELMs are expected to be the dominant source in ITER. W impurity concentration in the plasma core can dramatically degrade its performance and lead to potentially damaging disruptions. Understanding the physics of the target $\mathrm{W}$ source due to sputtering during ELMs and inter-ELMs is important and can be helped by experimental measurements with improved precision. It has been established that the ELMy target ion impact energy has a simple linear dependence with the pedestal electron temperature measured by Electron Cyclotron Emission (ECE). It has also been shown that Langmuir Probes (LP) ion flux measurements are reliable during ELMs due to the surprisingly low electron temperature. Therefore, in this paper, LP and ECE measurements in JET-ITER-Like-Wall (ILW) unseeded Type I ELMy H-mode experiments have been used to estimate the $\mathrm{W}$ sputtering flux from 
divertor targets in ELM and inter-ELM conditions. Comparison with similar estimates using W I spectroscopy measurements shows a reasonable agreement for the ELM and inter-ELM W source. The main advantage of the method involving LP measurements is the very high time resolution of the diagnostic $(\sim 10 \mu \mathrm{s})$ allowing very precise description of the $\mathrm{W}$ sputtering source during ELMs.

Keywords: tungsten sputtering, edge localized mode, magnetic confinement fusion

(Some figures may appear in colour only in the online journal)

\section{Introduction}

The ITER baseline scenario, with $500 \mathrm{MW}$ of DT fusion power and $\mathrm{Q}=10$, will rely on a Type I ELMy H-mode, see [1]. Partial divertor detachment with nitrogen $(\mathrm{N})$, neon $(\mathrm{Ne})$ or argon (Ar) impurity seeding will also be required to maintain the inter-ELM power load at managable level. Tungsten (W) is the material now decided for the divertor plasma-facing components from the start of plasma operations. Under the partially detached divertor conditions envisaged for ITER, W atoms sputtered from divertor targets during mitigated ELMs are expected to be the dominant impurity source. In ITER, W impurity concentration in the plasma core above $5 \times 10^{-5}$ can degrade fusion performance and may lead to potentially damaging disruptions, see [2]. Understanding the physics of $\mathrm{W}$ contamination in the plasma core is important and a primary input for this is the target $\mathrm{W}$ source mainly due to sputtering during ELMs.

The JET-ITER-Like-Wall (JET-ILW) [3] comprises a W divertor and beryllium $(\mathrm{Be})$ main chamber wall thus matching the material configuration planned for ITER. Due to the high energy threshold for physical sputtering of $\mathrm{W}$ by deuterium ions $\left(\mathrm{D}^{+}\right)$, the dominant $\mathrm{Be}$ ion species, $\mathrm{Be}^{2+}$, contributes to $\mathrm{W}$ sputtering in the divertor between ELMs (inter-ELM), see [4]. During ELMs, the experimental W sputtering yield due to $\mathrm{D}^{+}\left(Y_{\mathrm{D} / \mathrm{W}}\right)$ can be estimated providing that the target ion flux, the ion impact energy $\left(E_{\mathrm{i}}\right)$ and the ion impact angle $\left(\theta_{\mathrm{i}}\right)$ are known. It has already been established in [5] that electrons have low target temperature $\left(T_{\mathrm{e}}\right)$ in ELM conditions and can be repelled by biased Langmuir probes (LP) surfaces to allow reliable ion flux measurements, and also that the maximum $E_{\mathrm{i}}$ has a simple linear dependence with the maximum pedestal $T_{\mathrm{e}}$ $\left(T_{\mathrm{e}, \max }^{\mathrm{ped}}\right)$ measured by electron cyclotron emission (ECE). Therefore, experimental estimation of the $\mathrm{W}$ sputtering source using ECE and LP measurements seems feasible and has been attempted here.

A high power unseeded $\mathrm{H}-$ Mode discharge performed in JET-ILW (\#82237) where the W sputtering source has already been estimated using W I spectroscopy [6] has been used for this purpose. This experiment has been chosen for the slow $(\sim 10 \mathrm{~Hz})$ and large Type I ELMs allowing the use of slow W I spectroscopy ( $\sim 25 \mathrm{~Hz})$. The divertor configuration used in \#82237 features a vertical inner target with a horizontal outer target (OT), see figure 1. The present work has been focused on the use of ECE $T_{\mathrm{e}, \max }^{\mathrm{ped}}$ measurements and LP ion saturation current $\left(J_{\text {sat }}\right)$ measurements to estimate the W sputtering source on the horizontal OT in ELM and interELM conditions. This new method provides independent high time resolution $(\sim 10 \mu \mathrm{s})$ target measurements which can be compared to W I spectroscopy.

Before estimating the $\mathrm{W}$ sputtering source, it is essential to have an idea of $E_{\mathrm{i} \text { max }}$ in this experiment by using ECE pedestal temperature $\left(T_{\mathrm{e}}^{\mathrm{ped}}\right)$ measurements (section 2). Then, the experimental $\mathrm{W}$ sputtering yields due to $\mathrm{D}^{+}$and $\mathrm{Be}^{2+}$ in ELM and inter-ELM conditions can be estimated by calculating the different ion impact angle distributions (section 3).
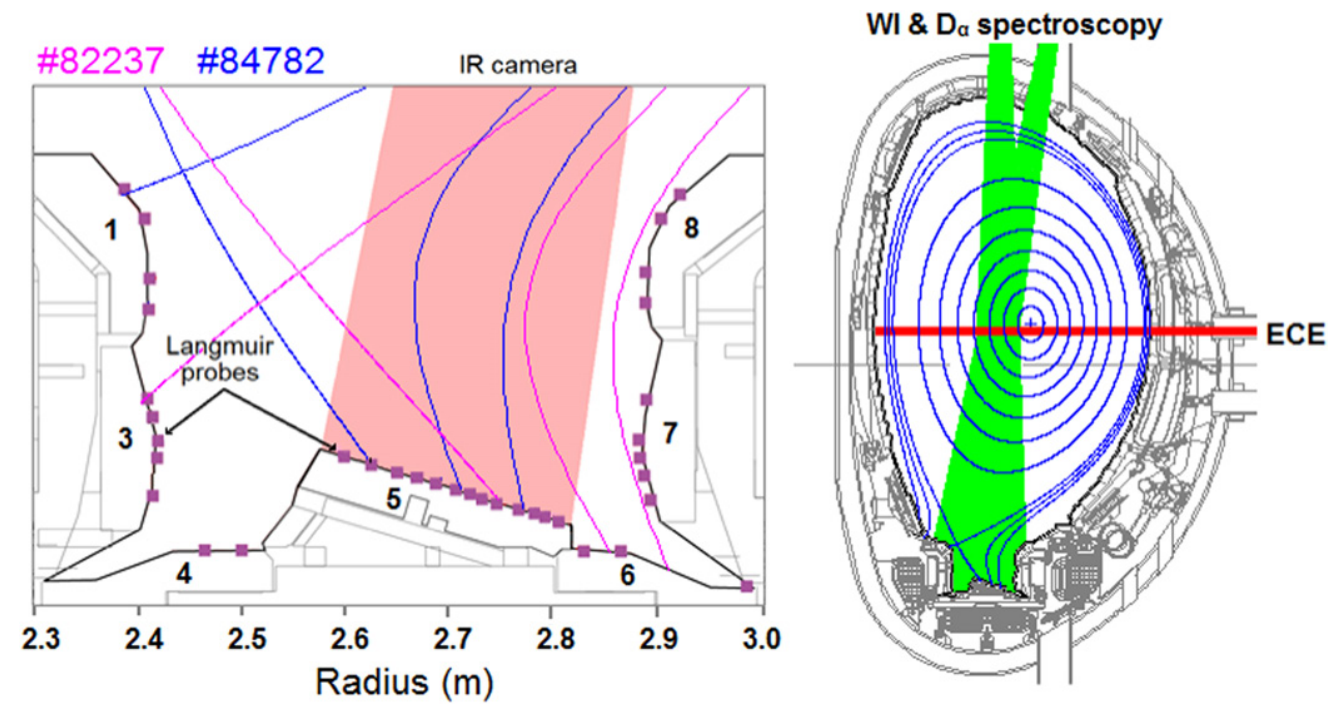

Figure 1. Left: positions of LP and IR camera line of sight in JET-ILW divertor with the different tile numbers. Right: magnetic equilibrium for \#82237 and 84782 at $13 \mathrm{~s}$ and position of W I, $\mathrm{D}_{\alpha}$ spectroscopy and ECE lines of sight in JET-ILW main chamber. 


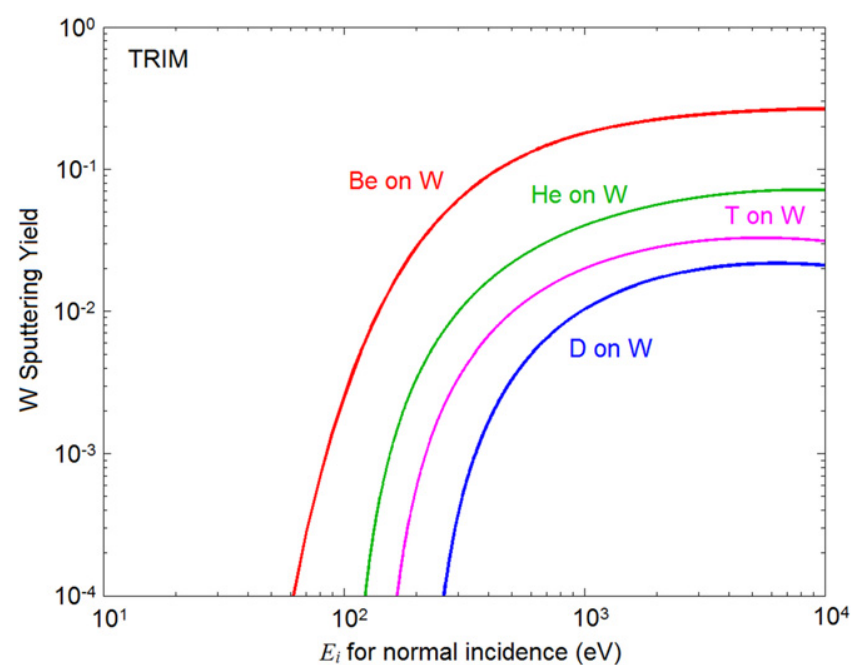

Figure 2. Curves of $\mathrm{W}$ sputtering yields at normal incidence due to Be in red, helium (He) in green, tritium (T) in magenta and deuterium (D) in blue.

Finally, OT W sputtering sources deduced from LP measurements have been compared to similar estimates made with W I spectroscopy [6] (section 4).

\section{2. $E_{\mathrm{i}}$ estimates during ELMs using ECE measurements}

As shown on the example in figure 2 obtained from TRIM [7], the W sputtering yield depends strongly on $E_{\mathrm{i}}$ for the different possible ion species striking the divertor targets. Previous studies [5] have shown that ELMy electrons have a surprisingly low target $T_{\mathrm{e}}$. As shown on figure 3, exponential fit of reconstructed ELMy $I-V$ characteristic from the strike point LP in a Type I ELMy H-mode discharge similar to \# 82237 yields $T_{\mathrm{e}} \sim 20-30 \mathrm{eV}$. This means that electrons can be repelled by LP biased surfaces during ELMs to allow ion flux measurements. Indeed, according to the 'Free-Streaming' model, it appears that during ELMS, electrons must transfer most of their parallel energy to the ions in order to maintain quasi-neutrality, see [8-10]. Therefore, LP ion saturation current $\left(J_{\text {sat }}\right)$ measurements in A.m ${ }^{-2}$ can be coupled to perpendicular heat flux density $\left(q_{\perp}\right)$ measurements in W.m ${ }^{-2}$ from infrared thermography (IR) to derive the sum of $E_{\mathrm{i}}$ with the electron impact energy $\left(E_{\mathrm{e}}\right)$ in $\mathrm{eV}$ as follows:

$$
E_{\mathrm{i}}+E_{\mathrm{e}}=\frac{q_{\perp}}{J_{\mathrm{sat}} \sin \theta_{\perp}}
$$

with $\theta_{\perp}$ the field line angle with the OT $\left(\sim 2-3^{\circ}\right.$ in JET-ILW). It has been previously verified [5] on several Type I ELMy H-mode discharges in JET-ILW that $E_{\mathrm{i}}+E_{\mathrm{e}}$ has a simple linear dependence with the $T_{\mathrm{e}, \max }^{\mathrm{ped}}$ such that:

$$
\max \left(E_{\mathrm{i}}+E_{\mathrm{e}}\right) \approx \alpha T_{\mathrm{e} \cdot \max }^{\mathrm{ped}}
$$

with $\alpha=5.23$ according to the 'Free-Streaming' model [8-10], see figure 4. Since the electron parallel energy is almost entirely transferred to the ions on their way to the

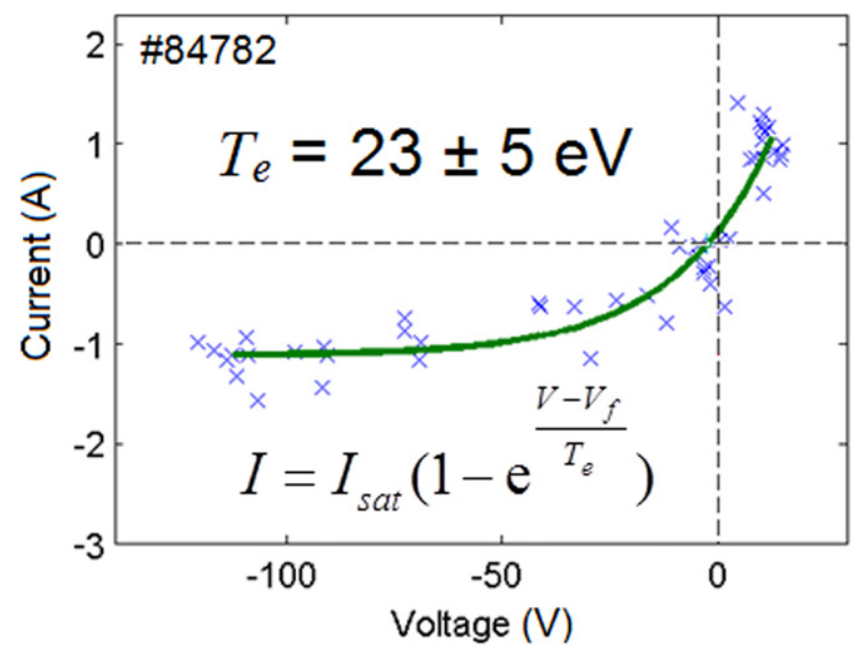

Figure 3. $I-V$ characteristic reconstruction obtained by cumulating $I$ and $V$ measurements taken by the LP in the peak ELMy ion flux of each ELM event over the discharge \#84782.

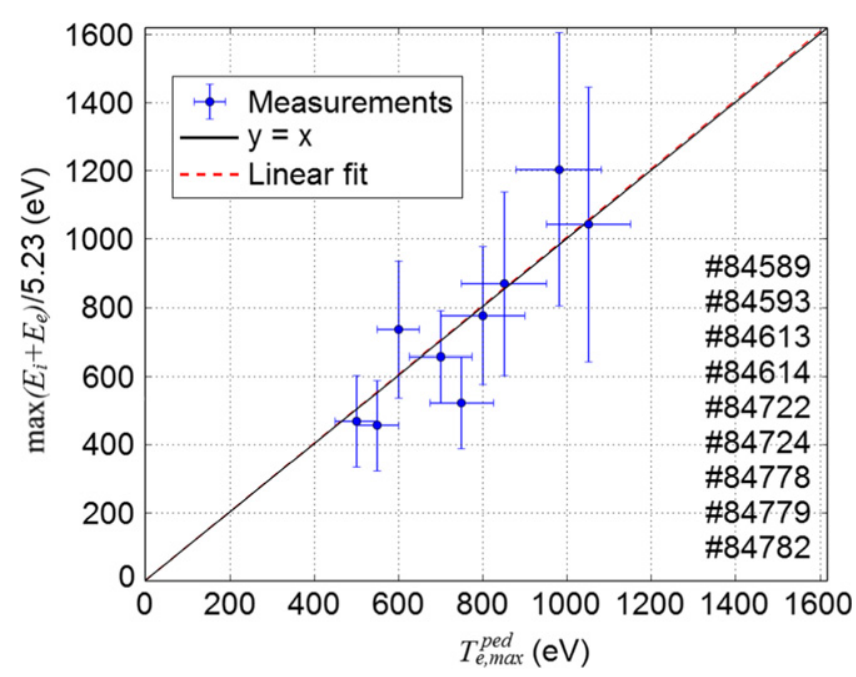

Figure 4. $\max \left(E_{\mathrm{i}}+E_{\mathrm{e}}\right) / 5.23$ as a function of $T_{\mathrm{e}, \max }^{\text {ped }}$ from coherent averaging of LP, IR and ECE measurements obtained in the discharges listed on the right.

target, the model assumes that the perpendicular electron energy $\left(E_{\mathrm{e}, \perp}\right)$ is the only component left in $E_{\mathrm{e}}$ such that:

$$
E_{\mathrm{e}}=E_{\mathrm{e}, \perp}=T_{\mathrm{e}}^{\mathrm{ped}} .
$$

Thus, $E_{\mathrm{i}, \max }$ during ELMs is:

$$
E_{\mathrm{i}, \max }=(\alpha-1) T_{\mathrm{e}, \max }^{\mathrm{ped}}=4.23 T_{\mathrm{e}, \max }^{\mathrm{ped}} .
$$

Here, the \#82237 JET-ILW Type I ELMy H-mode discharge already used to study the OT W sputtering source with W I spectroscopy [6] is considered. According to ECE measurement coherently averaged with the method described in [11], $T_{\mathrm{e}}^{\mathrm{ped}}$ max is $\sim 0.6 \mathrm{keV}$ here (figure $5(\mathrm{a})$ ), which means that following (4), $E_{\mathrm{i}, \max } \sim 2.5-3 \mathrm{keV}$. Mention has to be made that on ITER $T_{\mathrm{e}, \max }^{\text {ped }} \sim 5 \mathrm{keV}$ has been predicted [12] 

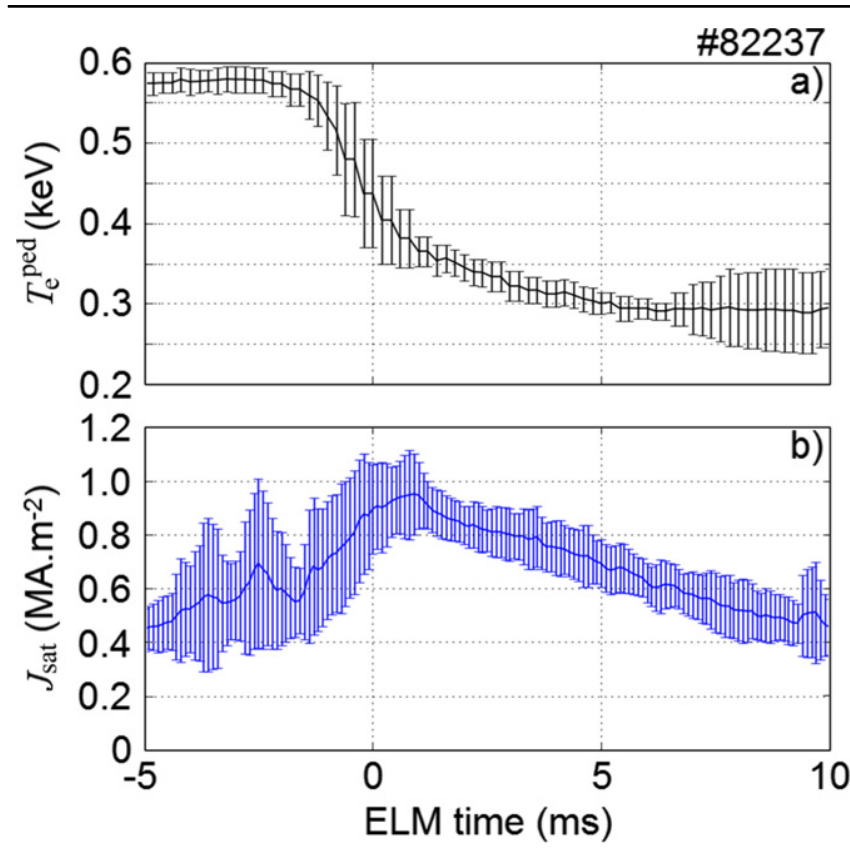

Figure 5. Coherently averaged (a) $T_{\mathrm{e}}^{\mathrm{ped}}$ with standard deviation measured by ECE and (b) strike point $J_{\text {sat }}$ over the cumulated ELM cycles of \#82237.

which means that $E_{\mathrm{i}, \max } \sim 20 \mathrm{keV}$ can be expected. Therefore, it is worth noting that the range of $E_{\mathrm{i}, \max }$ accessible in JET-ILW (2-4 keV according to [5]) allows similar W sputtering yields as those expected in ITER for the species considered in figure 2 since all the curves tend to saturate above $\sim 2-3 \mathrm{keV}$.

In inter-ELM conditions, it is considered, as in [4], that in a JET-ILW low $Z_{\text {eff }}$ plasma $\left(Z_{\text {eff }} \sim 1.1-1.3\right.$ here $)$ the ion impact energy $\left(E_{\mathrm{i}, \text { inter-ELM }}\right), T_{\mathrm{e}}$, and the target ion temperature $\left(T_{\mathrm{i}}\right)$ are such that:

$$
E_{\mathrm{i}, \text { inter }-\mathrm{ELM}} \approx 3 T_{\mathrm{e}}+2 T_{\mathrm{i}} .
$$

In \#82237, the maximum $T_{\mathrm{e}}$ from LP measurements is not higher than $\sim 30 \mathrm{eV}$ and if it is assumed that $T_{\mathrm{i}} \approx T_{\mathrm{e}}$, $E_{\mathrm{i} \text {,inter-ELM }}$ should not exceed $\sim 150 \mathrm{eV}$. For simplification, it is assumed in this work that both species of interest, namely $\mathrm{D}^{+}$and $\mathrm{Be}^{2+}$, have the same $E_{\mathrm{i}, \max }$ and $E_{\mathrm{i}, \text { inter-ELM }}$.

\section{Impact angle distribution in ELM and inter-ELM conditions}

The $\mathrm{W}$ sputtering yield due to $\mathrm{D}^{+}$and $\mathrm{Be}^{2+}\left(Y_{\mathrm{D} / \mathrm{W}}\right.$ and $Y_{\mathrm{Be} / \mathrm{W}}$ respectively) that we are trying to evaluate here also depend on the ion impact angle $\left(\theta_{\mathrm{i}}\right)$, as shown on figure 6 . Since the variation of $Y_{\mathrm{D} / \mathrm{w}}$ and $Y_{\mathrm{Be} / \mathrm{w}}$ with $\theta_{\mathrm{i}}$ can be up to an order of magnitude, it is crucial to estimate the distribution of this angle in ELM and inter-ELM conditions for $\mathrm{D}^{+}$and $\mathrm{Be}^{2+}$.

In inter-ELM conditions, $Y_{\mathrm{D} / \mathrm{W}}$ is neglected and only $Y_{\mathrm{Be} / \mathrm{W}}$ is considered. According to kinetic analytical calculations [13] considering a target plasma close to experimental inter-ELM conditions with Maxwellian distribution of energy

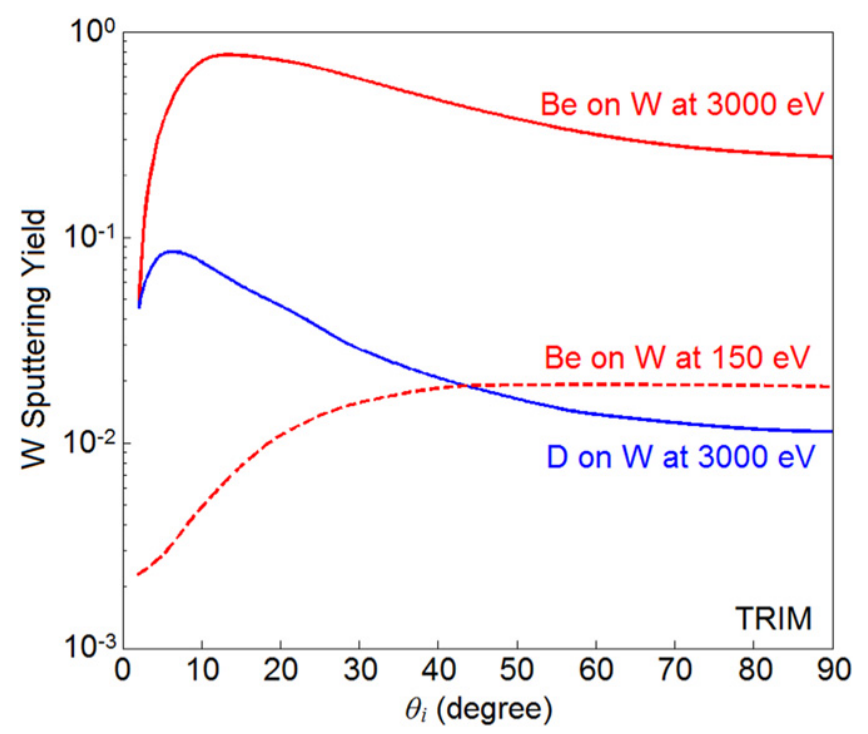

Figure 6. $\theta \mathrm{i}$ dependence of $\mathrm{W}$ sputtering yield due to $\mathrm{D}^{+}$(blue curve) and $\mathrm{Be}^{2+}$ (red curves) for $E_{\mathrm{i}}=150 \mathrm{eV}$ (dashed) and $E_{\mathrm{i}}=3000 \mathrm{eV}$ (plain).

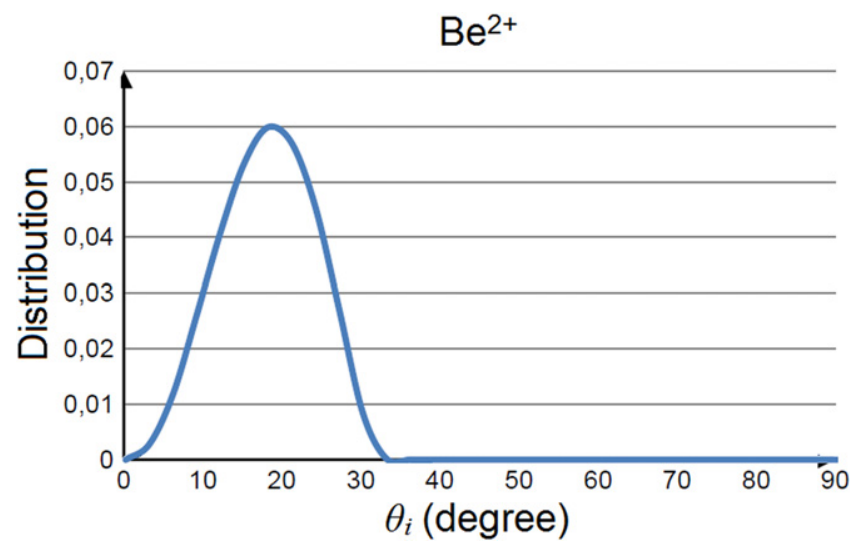

Figure 7. Distribution of $\mathrm{Be}^{2+}$ OT impact angle given by analytical kinetic calculations [13] with $T_{\mathrm{e}}=T_{\mathrm{i}}=30 \mathrm{eV}, n_{\mathrm{e}} \sim 10^{19} \mathrm{~m}^{-3}$, $\theta_{\perp}=3^{\circ}$ and $B=3 \mathrm{~T}$.

for ions and electrons, $T_{\mathrm{e}}=T_{\mathrm{i}}=30 \mathrm{eV}$, electron density $n_{\mathrm{e}}=10^{19} \mathrm{~m}^{-3}$, magnetic field $B=3 \mathrm{~T}$ and $\theta_{\perp}=3^{\circ}$, the distribution of inter-ELM $\theta_{\mathrm{i}}$ should reach its maximum around $\sim 20^{\circ}$ for $\mathrm{Be}^{2+}$, see figure 7 .

During ELMs, despite $E_{\mathrm{i} \text {,max }} \sim 3 \mathrm{keV}$ in average in JETILW, the target $T_{\mathrm{e}}$ remains close to the inter-ELMs level. As in the example shown in figure $3, T_{\mathrm{e}}$ in \#82237 should not be above $\sim 30 \mathrm{eV}$ in ELMy conditions. However, the higher number of ionization per $\mathrm{D}_{\alpha}$ photon required to match LP ion flux measurements in [5], when the recycling coefficient is assumed to be around unity, is an evidence of higher $n_{\mathrm{e}}$ of the order of $\sim 10^{20} \mathrm{~m}^{-3}$ during ELMs. Also, according to the 'Free-Streaming' model [8-10], the high energy ELMy ions have a very narrow energy distribution when they reach the target. Kinetic analytical calculations [13] with these conditions and the same $B$ and $\theta_{\perp}$ as the inter-ELM case, yield a distribution of $\theta_{\mathrm{i}}$ reaching its maximum around $22^{\circ}$ for $\mathrm{D}^{+}$ and $25^{\circ}$ for $\mathrm{Be}^{2+}$, see figures $8(\mathrm{a})$ and (b). The small 

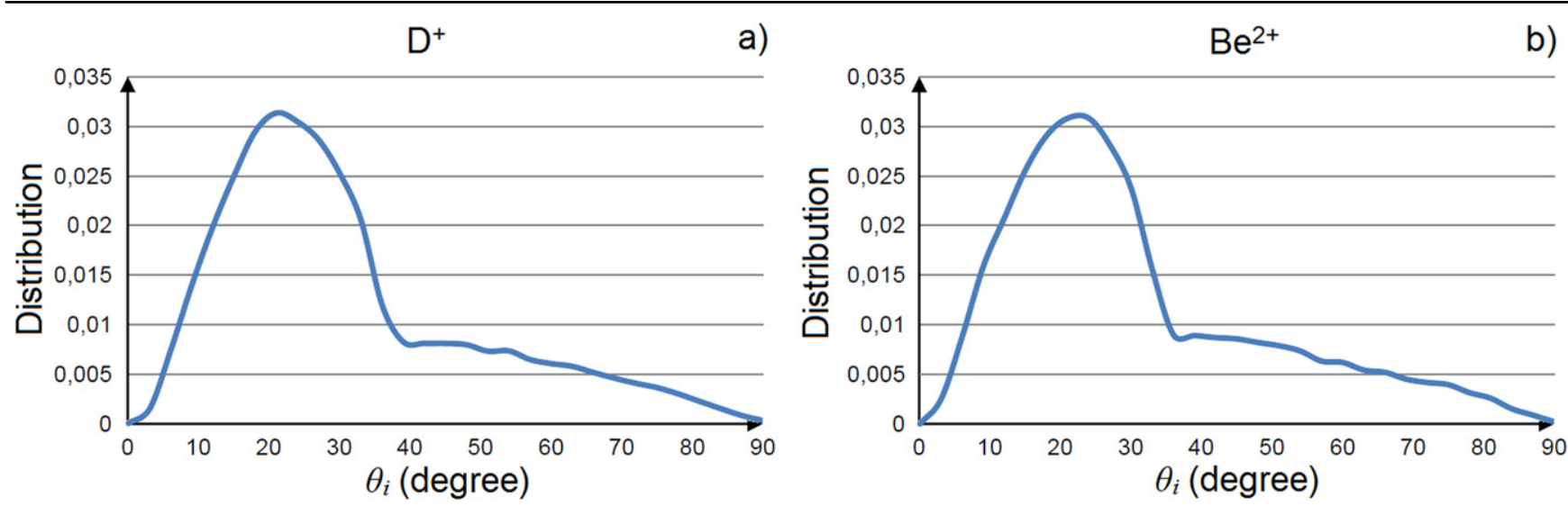

Figure 8. Distribution of (a) $\mathrm{D}^{+}$and (b) $\mathrm{Be}^{2+} \mathrm{OT}$ impact angle given by analytical kinetic calculations [13] with $T_{\mathrm{e}}=30 \mathrm{eV}, E_{\mathrm{i}}=3 \mathrm{keV}$, $n_{\mathrm{e}}=10^{20} \mathrm{~m}^{-3}, \theta_{\perp}=3^{\circ}$ and $B=3 \mathrm{~T}$.

oscillations in the distribution of $\theta_{\mathrm{i}}$ are due to limitations in the number of test particles used in the kinetic calculations. The level of precision of the calculated distribution is however sufficient to allow estimation of the sputtering yield.

The small difference between ELM and inter-ELM conditions in terms of $\theta_{\mathrm{i}}$ distribution suggests that the latter is more influenced by the ion gyration than the sheath effects. Indeed, if the electric field of the Debye sheath had a strong effect, the $\theta_{\mathrm{i}}$ distribution should be much closer to $90^{\circ}$. As shown in figure 6 , shallow $\theta_{\mathrm{i}}$ around $\sim 20^{\circ}$ at high $E_{\mathrm{i} \text {,max }}$ are associated with significant $\mathrm{W}$ sputtering yields.

\section{Estimation of $W$ impurity sputtering in ELM and inter-ELM conditions}

Given the $\theta_{\mathrm{i}}$ distribution obtained previously in inter-ELM, the average $Y_{\mathrm{Be} / \mathrm{W}}$ should be around $\sim 0.01$ in these conditions, according to figure 6. During ELMs, the average $\mathrm{W}$ sputtering yield due to $\mathrm{Be}^{2+}$ and $\mathrm{D}^{+}$should reach $Y_{\mathrm{Be} / \mathrm{W}} \sim 0.6$ and $Y_{\mathrm{D} / \mathrm{w}} \sim 0.03$ respectively, considering the ELMy $\theta_{\mathrm{i}}$ distributions previously calculated. Since the Be concentration in the impinging ion flux is expected to be around $\sim 0.5 \%$ in unseeded JET-ILW Type I ELMy H-mode experiments [4], all the required parameters are known to estimate the W sputtering source on the OT by using LP $J_{\text {sat }}$ measurements.

The $\mathrm{W}$ sputtering flux due to the cumulated effect of $\mathrm{D}^{+}$ and $\mathrm{Be}^{2+}$ in ELM and inter-ELM has already been estimated in [6], using W I spectroscopy. However, despite the known $T_{\mathrm{e}}$ dependence of the number of ionizations per emitted photon from W I discussed in [14], the remaining uncertainties related to possible $n_{\mathrm{e}}$ dependence and $T_{\mathrm{e}}$ measurements still leave some room for a significant error. Thus, an uncertainty on the number of ionizations per photon and consequently on the estimated $\mathrm{W}$ sputtering source remains. Moreover, the mirror-link system viewing the OT and analyzing the light in three wavelength ranges with CzerneyTurner spectrometers [15] has a time resolution limited to $40 \mathrm{~ms}$ which is slow compared to the typical $\sim 1 \mathrm{~ms}$ duration of the Type I ELM events studied here. Typical measurements made during ELMy H-mode discharges with this diagnostic in JET-ILW cumulate light from several ELMs and inter-ELMs and only very slow Type I ELMs like in \#82237 can be isolated once or twice over the duration of the experiment $(\sim 5 \mathrm{~s})$. Thus, these measurements cannot be coherently averaged and ELMs must be very similar all along the discharge in order to consider ELMy W I spectroscopy data representative of a typical ELM.

Since LP $J_{\text {sat }}$ measurements are accurate during ELMs and inter-ELMs [5], independent estimation of the W sputtering source based on this signal provides an opportunity for a comparison with the W I spectroscopy technique. This new method involving the LP has the advantage of not relying on any assumptions on atomic physics and benefits from the high time resolution of the $J_{\text {sat }}$ signal $(\sim 10 \mu \mathrm{s})$ required during ELMs and allowing coherent averaging like in figure 5(b). However, it assumes that relation (4) is true for any Type I ELMy H-mode and that $E_{\mathrm{i}, \max }$ remains constant at $\sim 3 \mathrm{keV}$ over the entire OT for a duration of $\Delta t_{\mathrm{ELM}} \sim 1 \mathrm{~ms}$ in average (duration of $T_{\mathrm{e}}^{\text {ped }}$ drop in figure 5(a)) in each ELM event.

The $\mathrm{W}$ sputtering flux densities $\Gamma_{\mathrm{W}, \text { ELM }}$ and $\Gamma_{\mathrm{W} \text {,inter-ELM }}$ in $\mathrm{m}^{-2} \mathrm{~s}^{-1}$ due to ELM and inter-ELM ion flux respectively have been calculated as follows:

$$
\begin{aligned}
& \Gamma_{\mathrm{W}, \mathrm{ELM}} \approx \frac{J_{\mathrm{sat}, \mathrm{ELM}}}{e} \sin \left(\theta_{\perp}\right)\left(Y_{\mathrm{D} / \mathrm{W}}\left(3 \mathrm{keV}, 22^{\circ}\right)\right. \\
&\left.+0.005 Y_{\mathrm{Be} / \mathrm{W}}\left(3 \mathrm{keV}, 25^{\circ}\right)\right) \Delta t_{\mathrm{ELM}} f_{\mathrm{ELM}}, \\
& \Gamma_{\mathrm{W}, \text { inter-ELM }} \approx \frac{0.005 J_{\mathrm{sat}, \text { inter }-\mathrm{ELM}}}{e} \sin \left(\theta_{\perp}\right) \\
& \times Y_{\mathrm{Be} / \mathrm{W}}\left(150 \mathrm{eV}, 20^{\circ}\right)
\end{aligned}
$$

With $J_{\text {sat,ELM }}, J_{\text {sat,inter-ELM }}$ and $f_{\text {ELM }}$ the ELMy $J_{\text {sat }}$, the inter-ELM $J_{\text {sat }}$ both measured in A.m ${ }^{-2}$ by the LP and the ELM frequency in $\mathrm{s}^{-1}$ respectively. Thus, the ELM and interELM experimental perpendicular ion flux density profiles shown on figure 9(a) and obtained from the LP allow the estimated $\Gamma_{\mathrm{W}, \mathrm{ELM}}$ and $\Gamma_{\mathrm{W} \text {,inter-ELM }}$ profiles shown on figure 9(b) for the discharge \#82237.

Previous $\Gamma_{\mathrm{W}, \mathrm{ELM}}$ and $\Gamma_{\mathrm{W} \text {,inter-ELM profiles deduced from }}$ W I spectroscopy [6] are also shown on figure 9(b) for 

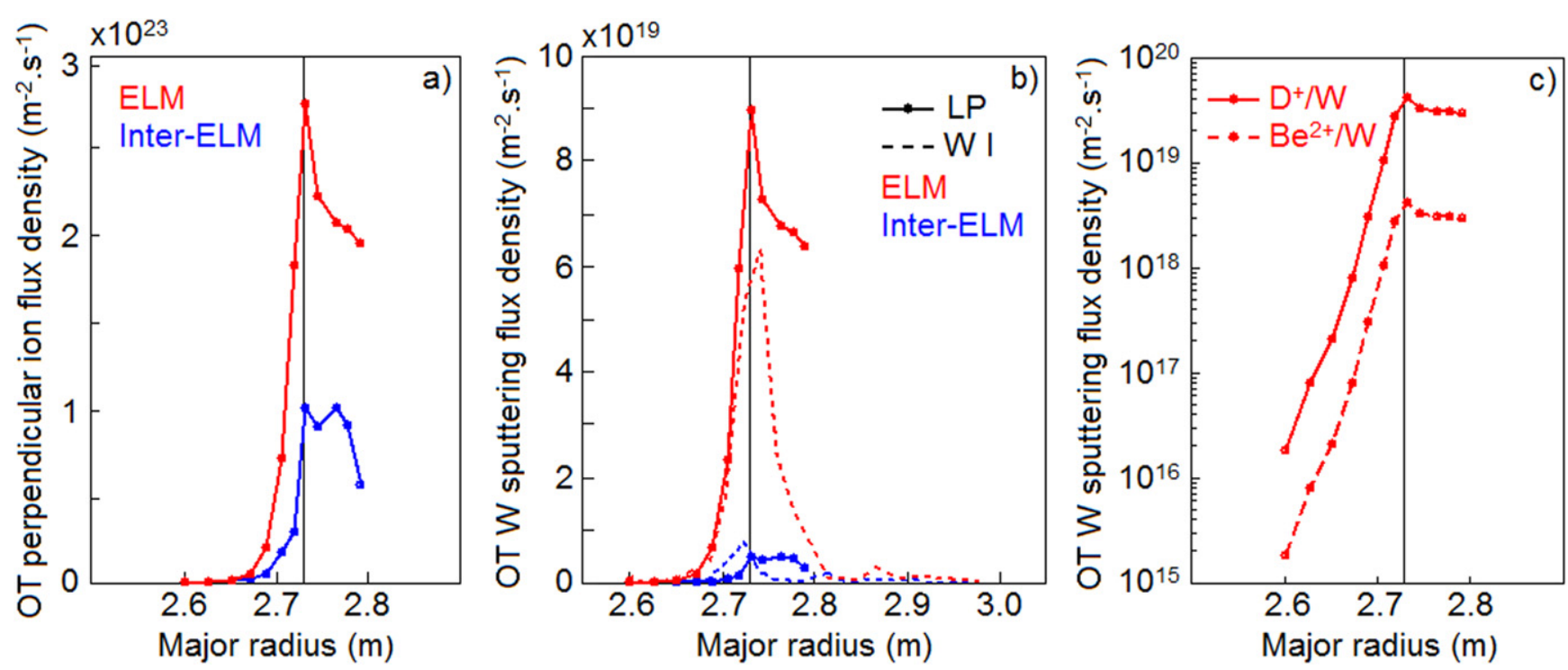

Figure 9. (a) OT perpendicular ion flux density profile from LP, (b) OT total W sputtering flux density profile from WI spectroscopy (dashed curves) and LP (plain curves) due to ELM (red curves) and inter-ELM (blue curves) and (c) contribution from $\mathrm{D}^{+}$(plain curve) and Be ${ }^{2+}$ (dashed curve) to OT W sputtering flux density profile.

Table 1. ELMy W sputtering fluence and inter-ELM W sputtering flux on OT.

\begin{tabular}{lcc}
\hline Method & W I spectroscopy & LP \\
\hline $\begin{array}{l}\text { ELMy W fluence } \\
(\text { atoms.ELM }\end{array}$ & $5.7 \times 10^{18}$ & $11 \times 10^{18}$ \\
\begin{tabular}{l} 
Inter-ELM W flux (atoms. $\left.{ }^{-1}\right)$ \\
\hline
\end{tabular} & $6.3 \times 10^{18}$ & $5.9 \times 10^{18}$ \\
\hline
\end{tabular}

comparison. Both flux density profiles have less than $30 \%$ discrepancy in magnitude. OT W sputtering fluence per ELM and OT inter-ELM W sputtering flux from both methods are provided in table 1. Discrepancies between integrated amounts obtained from both methods of measurements do not exceed a factor 2 during ELMs and are below $10 \%$ in interELM. The order of magnitude of the OT W source in discharge \#82237 with $\sim 10^{19} \mathrm{~W}$ atoms per ELM and $\sim 10^{19} \mathrm{~s}^{-1}$ $\mathrm{W}$ flux during inter-ELM is confirmed by both techniques. Therefore, given the assumptions and approximations made to get these estimations, this level of agreement is reasonably good.

The comparison of $\mathrm{W}$ sputtering flux density profiles due to ELMy $\mathrm{D}^{+}$and $\mathrm{Be}^{2+}$ on the OT shown on figure 9(c) indicates that $\mathrm{D}^{+}$during ELM is the main contributor to the W source in JET-ILW unseeded Type I ELMy H-modes. Indeed, in these conditions, the $\mathrm{Be}^{2+}$ contribution is lower by an order of magnitude because of the small Be concentration in the impinging ion flux of $\sim 0.5 \%$, see [4].

\section{Conclusions}

Mitigated Type I ELMs expected in ITER for the baseline scenario with $500 \mathrm{MW}$ of fusion power and $\mathrm{Q}=10$, are expected to be the dominant source of W. Very small amounts of $\mathrm{W}$ will be tolerated in the plasma core to ensure good performance [2]. Therefore, understanding the physics of the target W source due to sputtering during ELMs and interELMs is important and can be helped by experimental measurements with improved precision.

Previous studies [5] coupling IR and LP measurements in JET-ILW H-mode experiments with ITER relevant ELM energy drop have shown that $E_{\mathrm{i} \text {,max }}$ during ELMs is in the range $2-4 \mathrm{keV}$ for $\mathrm{D}^{+}$and has a simple linear dependence on $T_{\mathrm{e}, \max }^{\mathrm{ped}}$. Saturation of the ion current measured by the LP during ELMs is possible thanks to the surprisingly low $T_{\mathrm{e}}$ of the order of the inter-ELM conditions which is consistent with the predictions from the 'Free-Streaming' model for the description of parallel ELM transport [8-10]. According to the model, electrons have to transfer most of their parallel energy to the ions on their way to the target to maintain the quasi-neutrality of the ELM filaments. The remaining low energy ELMy electrons are therefore easy to repel by the biased LP at the targets, making the ion flux measurement possible during ELMs.

Consequently, providing that the distribution of $\theta_{\mathrm{i}}$ is known, ECE $T_{\mathrm{e}}^{\text {ped }}$ measurements and LP $J_{\text {sat }}$ measurements can be used to estimate the $\mathrm{W}$ sputtering source due to $\mathrm{D}^{+}$ and $\mathrm{Be}^{2+}$ in inter-ELM and ELM conditions. The unseeded Type I ELMy H-mode discharge \#82237, where the W sputtering source has already been estimated using W I spectroscopy [6], has been used here for this purpose.

$\mathrm{W}$ sputtering flux density profiles obtained from W I spectroscopy and LP measurements differ by less than $\sim 30 \%$ in magnitude an confirm the order of magnitude of the $\mathrm{W}$ sputtering source with roughly $\sim 10^{19}$ atoms per ELM and $\sim 10^{19}$ atoms.s $^{-1}$ in inter-ELM in \#82237. Since the ELM frequency in this discharge is $\sim 10 \mathrm{~Hz}$, the ELM W source is dominant by an order of magnitude over the inter-ELM W source.

Comparison of $\mathrm{W}$ sputtering flux density profiles due to ELMy $\mathrm{D}^{+}$and $\mathrm{Be}^{2+}$ on the OT indicates that $\mathrm{D}^{+}$during 
ELMs is the main contributor to the $\mathrm{W}$ source. The $\mathrm{Be}^{2+}$ contribution is lower by an order of magnitude because of the small $\mathrm{Be}$ concentration in the impinging ion flux of $\sim 0.5 \%$ in JET-ILW unseeded Type I ELMy H-modes, see [4].

Given the uncertainty on the number of ionizations per photon in W I spectroscopy and the approximation that $E_{\mathrm{i} \text {,max }}$ remains constant at $\sim 3 \mathrm{keV}$ over the entire OT during each ELM event, OT W sputtering source estimates from both methods are in reasonably good agreement.

The very high time resolution $(\sim 10 \mu \mathrm{s})$ of the LP $J_{\text {sat }}$ measurements is a strong advantage which should allow the use of this new technique to estimate the $\mathrm{W}$ sputtering source in other conditions where ELMs are usually too fast for W I spectroscopy like seeded H-mode discharges or ELM pacing experiments.

\section{Acknowledgments}

This work has been carried out within the framework of the EUROfusion Consortium and has received funding from the Euratom research and training programme 2014-2018 under grant agreement No 633053 and the MSMT INGO grant LG14002. IST activities also received financial support from 'Fundação para a Ciência e Tecnologia' through project
UID/FIS/50010/2013. The views and opinions expressed herein do not necessarily reflect those of the European Commission.

\section{References}

[1] Loarte A et al 2014 Nucl. Fusion 54033007

[2] Kallenbach A et al 2005 Plasma Phys. Control. Fusion 47 B207-22

[3] Matthews G F et al 2011 Phys. Scr. 2011014001

[4] Brezinsek S et al 2013 J. Nucl. Mater. 463 S11-21

[5] Guillemaut C et al 2015 Plasma Phys. Control. Fusion 57 085006

[6] van Rooij G J et al 2013 J. Nucl. Mater. 438 (Suppl) S42-7

[7] Eckstein W et al 2002 Calculated sputtering,reflection and range values IPP-Report IPP 9/132 (Garching: MaxPlanck-Institut für Plasmaphysik)

[8] Fundamenski W et al 2006 Plasma Phys. Control. Fusion 48 109-56

[9] Eich T et al 2009 J. Nucl. Mater. 390-1 760-3

[10] Moulton D et al 2013 Plasma Phys. Control. Fusion 55 085003

[11] Pitts R A et al 2003 Nucl. Fusion 43 1145-66

[12] Sugihara M et al 2003 Plasma Phys. Control. Fusion 45 L55-62

[13] Borodkina I et al 2015 Russ. Phys. J. 4 9-15

[14] Dux R et al 2009 J. Nucl. Mater. 390-1 858

[15] Meigs A et al 2010 Rev. Sci. Instrum. 81 10E532 